\title{
Incidence and location of bone metastases in breast cancer
}

\author{
Monica Cirstoiu ***, Octavian Munteanu***, Oana Bodean*, Florina Paulet***, \\ Bogdan Șerban ****, Bogdan Cretu***, Cătălin Cirstoiu**** \\ * "Carol Davila" University of Medicine and Pharmacy, Bucharest, Romania \\ $* *$ Department of Obstetrics and Gynaecology, University Emergency Hospital, Bucharest, Romania \\ ***Department of Orthopedics and Traumatology, University Emergency Hospital, Bucharest, Romania
}

Correspondence to: Octavian Munteanu, MD, PhD, Department of Obstetrics and Gynaecology, University Emergency Hospital, Bucharest, 169 Splaiul Independenței Street, District 5, Bucharest, Romania, Phone: 02131805 19, int. 521, Mobile phone: +40722650092 , E-mail: octav_munteanu@yahoo.com

\begin{abstract}
This report represents an analysis of 19 confirmed cases of breast cancer throughout a period of 14 months (January 2018 - February 2019), in which we assessed the incidence and sites of osseous metastases, in correlation with their histopathologic classification. This retrospective analysis is based on medical imaging techniques (X-ray radiography and nuclear medicine functional imaging).

Keywords: osseous metastases, breast cancer, incidence
\end{abstract}

\section{Introduction}

During 2018, in Romania, a total of 9629 new cases of breast cancer were documented [1], ranking this type of neoplasia as the 2nd among the most common types of cancer in women.

The process of metastasizing involves loss of intercellular cohesion, cell migration, angiogenesis, access to systemic circulation, survival in circulation, evasion of local immune responses, and growth at distant organs $[2,3]$.

The bone is the third most frequent site of metastasis, after lung and liver [3]. Prostate and breast cancer $(B C)$ are responsible for the majority of the skeletal metastases (up to $70 \%$ ) [4]. This reflects both the high incidence and relatively long clinical course of these tumors.

Numerous studies have documented factors that increase the relative risk for breast cancer [5]:

- Age

- Personal history of breast cancer

- History of atypical hyperplasia (ductal or lobular) on past biopsies

- Inherited genetic mutations

- First-degree relatives with breast or ovarian cancer diagnosed at an early age

- Early menarche (age > 12 years)

- Late onset of menopause (age > 55 years)

- Late age at first live birth (>30) 
- Alcohol consumption

- Recent oral contraceptive use

- Use of hormone therapy

- Personal history of endometrial, ovarian or colon cancer.

Some malignant tumors demonstrate a greater predilection for osseous involvement. In women, carcinoma of the breast is responsible for almost $70 \%$ of all metastatic lesions, the remaining $30 \%$ being mainly due to carcinomas of the thyroid, uterus, and kidney [6].

In particular, bone metastases can be confused with osteoporosis or Paget's disease. Osteoporosis is common in the elderly, and can be accelerated by certain anticancer treatments, premature menopause secondary to ovary ablation or cytotoxic chemotherapy in patients with breast cancer $[7,8]$, tamoxifen use in premenopausal patients with breast cancer [9].

\section{Materials and methods}

A retrospective analysis of all the patients with histologically proven carcinoma of the breast attending the Department of Obstetrics and Gynecology of the University Emergency Hospital in Bucharest between 1 January 2018 and 28 February 2019 has been made. Defined groups have been selected to enable the analysis of incidence, as well as the most common site of bone metastasis. The patients were divided into groups based on the histopathological type of the breast tumor and on the imaging technique used to determine the stage of the neoplasia preoperatively. All analyses were conducted using SPSS version 19.

\section{Results}

30 patients, with a mean age of 53.2 years old, were admitted with the diagnosis of breast tumor in the Department of Obstetrics and Gynecology of the University Emergency Hospital, in Bucharest. 63.33\% ( $n=19)$ of the patients were diagnosed with breast cancer. Out of these cases, only 2 were documented with osseous metastases. One patient had a lytic lesion located in the distal tibia, which underwent surgery - intramedullary nailing (see Fig. 1). The other patient had a blastic lesion located on the vertebral body (see Fig. 2) and also hepatic dissemination (see Fig. 3).
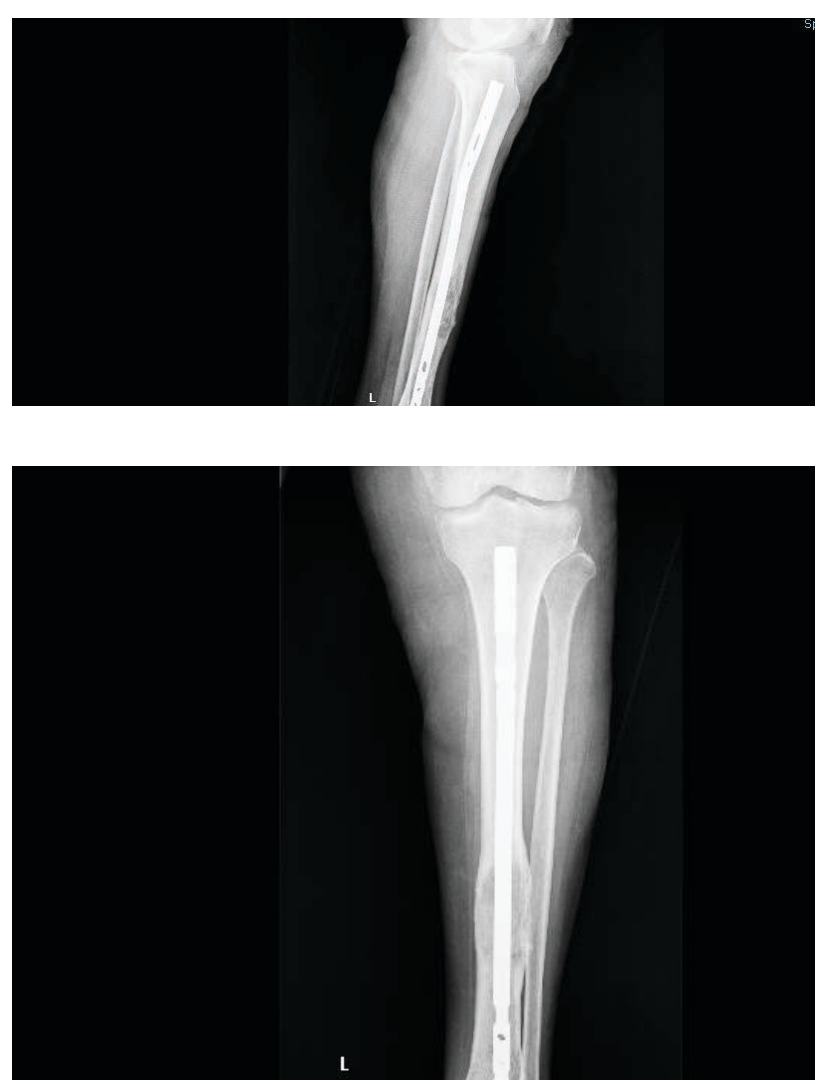

Fig. 1 Distal tibia lytic lesion secondary to a breast cancer early intramedullary nailing

The types of lesions encountered in bone metastases can be lytic, blastic, or mixed depending on the type of cancer. Lytic metastases form when bone resorption is present like in breast cancer, thyroid, renal and gastrointestinal cancers. It is mixed, when a patient has both osteolytic and osteoblastic lesion components (e.g. gastrointestinal cancers, breast cancer). 


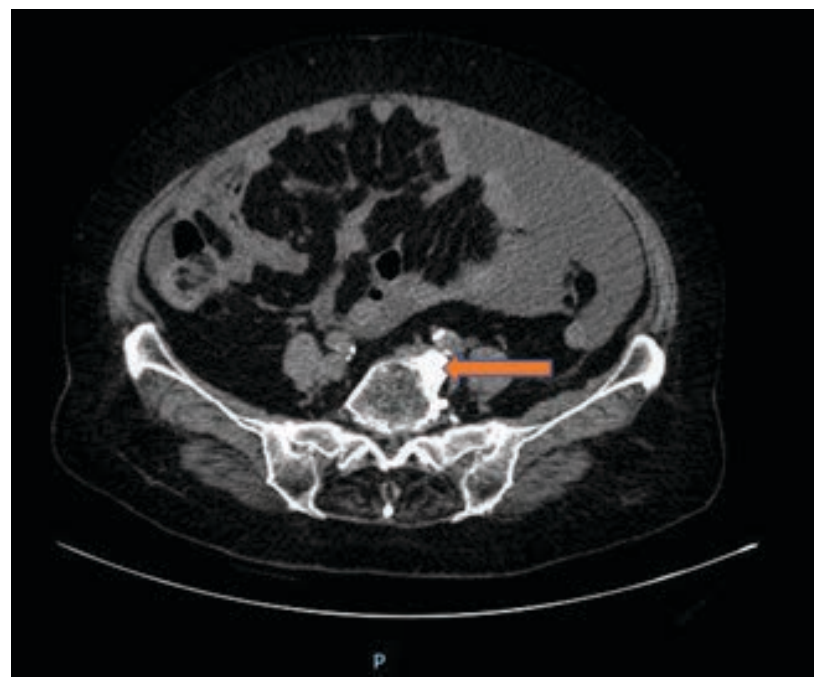

Fig. 2 Blastic lesion secondary to a breast cancer, located on the vertebral body

Lymphovascular invasion was detected in $26.32 \%$ of the cases $(n=5)$ diagnosed with breast cancer, whereas pulmonary and liver metastases were each observed in one case.
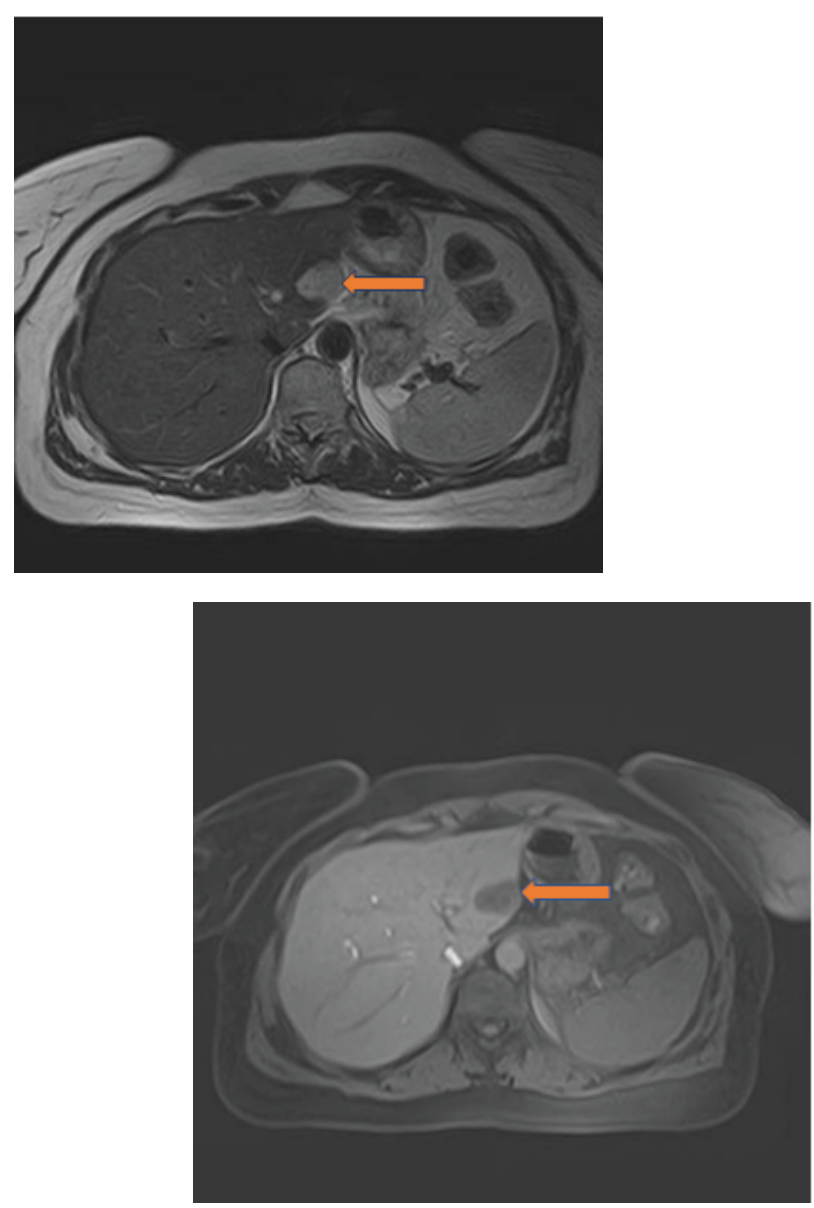

Fig. 3 Pseudonodular secondary hepatic dissemination due to breast cancer
Most of the cases were identified during stage IA (14 cases), followed by IIA (4 cases), and IIIA ( 1 case).

\section{Conclusions}

Osseous metastases are a sign of disseminated disease and foretell a shortterm prognosis in cancer patients. The bone metastases have an important impact on patient's quality of life so, new strategies are necessary to prevent skeletal disease and palliate established skeletal events.

A multidisciplinary approach should include medical oncologist, radiotherapist, pain control team, intervention radiologist, endocrinologist, orthopedic surgeon, and psychologist.

\section{Disclosure}

None of the authors has a conflict of interest.

All the authors have participated equally in the development of this study.

\section{Conflict of Interest statements}

Authors state no conflict of interest.

\section{Informed Consent and Human and Animal Rights statements \\ Informed consent has been obtained from all individuals included in this study.}

\section{Authorization for the use of human subjects}

Ethical approval: The research related to human use complies with all the relevant national regulations, institutional policies, is in accordance with the tenets of the Helsinki Declaration, and has been approved by the authors' institutional review board or equivalent commite

\section{References}

1. http://gco.iarc.fr/today/data/factsheets/populations/642-romania-fact-sheets.pdf.

2. van Zijl F, Krupitza G, Mikulits W. Initial steps of metas- 
tasis: cell invasion and endothelial transmigration. $\mathrm{Mu}-$ tation Research/Reviews in Mutation Research. 2011; 728:(1-2),23-3.

3. Chambers AF, Groom AC, MacDonald IC. Metastasis: dissemination and growth of cancer cells in metastatic sites. Nature Reviews Cancer. 2002; 2(8),563.

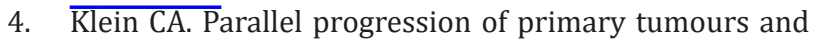
metastases. Nature Reviews Cancer. 2009; 9(4),302.

5. McPherson K, Steel C, Dixon JM. ABC of breast diseases: breast cancer-epidemiology, risk factors, and genetics. BMJ: British Medical Journal. 2000; 321(7261),624.

6. Abrams HL. Skeletal metastases in carcinoma. Radiology. 1950; 55:534-538,3.

7. Rivkees A, Crawford J. The relationship of gonadal activity and chemotherapy-induced gonadal damage. JAm Med Assoc. 1988; 259:2123-5.

8. Saarto $\mathrm{T}$ et al. Chemical castration induced by adjuvant cyclophosphamide, methotrexate, and fluorouracil chemotherapy causes rapid bone loss that is reduced by c1odronate: a randomized study in premenopausal breast cancer patients. J Clin Oncol. 1997; 15:1341-7.

9. Powles $\mathrm{T}$ et al. The effect of tamoxifen on lumbar bone mineral density in pre- and post-menopausal women. J Clin Oncol. 1996; 14:78-84. 